\title{
Article
}

\section{Study of roughness effect in Fe and Co thin films prepared by plasma magnetron sputtering}

Belusky, M., Lepadatu, Serban, Naylor, J. and Vopson, M.M.

Available at http://clok.uclan.ac.uk/29660/

Belusky, M., Lepadatu, Serban ORCID: 0000-0001-6221-9727, Naylor, J. and Vopson, M.M. (2019) Study of roughness effect in Fe and Co thin films prepared by plasma magnetron sputtering. Physica B: Condensed Matter, 574 . p. 411666. ISSN 0921-4526

It is advisable to refer to the publisher's version if you intend to cite from the work. http://dx.doi.org/10.1016/j.physb.2019.411666

For more information about UCLan's research in this area go to http://www.uclan.ac.uk/researchgroups/ and search for <name of research Group>.

For information about Research generally at UCLan please go to http://www.uclan.ac.uk/research/

All outputs in CLoK are protected by Intellectual Property Rights law, including Copyright law. Copyright, IPR and Moral Rights for the works on this site are retained by the individual authors and/or other copyright owners. Terms and conditions for use of this material are defined in the policies page.

\section{CLoK}

Central Lancashire online Knowledge www.clok.uclan.ac.uk

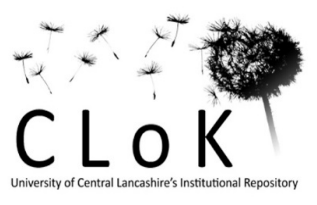




\title{
Study of roughness effect in Fe and Co thin films prepared by plasma magnetron sputtering
}

\author{
M. Belusky ${ }^{1}$, S. Lepadatu ${ }^{2}$, J. Naylor ${ }^{3}$, M.M. Vopson ${ }^{1}$ \\ ${ }^{1}$ University of Portsmouth, School of Mathematics and Physics, Portsmouth PO1 3QL, UK \\ ${ }^{2}$ University of Central Lancashire, Jeremiah Horrocks Institute for Mathematics, Physics and Astronomy, \\ Preston PR1 2HE, UK \\ ${ }^{3}$ Kurt J Lesker Ltd, 15/16 Burgess Road, Hastings, East Sussex, TN35 4NR, UK
}

\begin{abstract}
We report the experimental and theoretical study of a substrate roughness surface induced magnetic anisotropy in thin films of Fe and $\mathrm{Co}$. The experimental results confirm previously reported data on NiFe thin films and indicate that rough substrates increase the magnetic coercive fields in magnetic thin films. This effect is most prominent in films of thickness comparable to the surface roughness values and materials of small volume magnetic anisotropy. We determined the coercive field of $15 \mathrm{~nm}$ Fe thin film sample deposited onto rough PVDF to be $256 \mathrm{Oe}$, which is more than doubled the value of the coercive field of $15 \mathrm{~nm}$ Fe films coated under identical conditions, onto smooth $\mathrm{Si}$ substrates. The effect is visible for Co films but weaken by its increased volume magneto-crystalline anisotropy. These results are important for applications based on magnetic thin film where the magnetic properties could be adjusted via substrate roughness engineering
\end{abstract}

Keywords: Plasma sputtering, Thin film, Roughness, Magnetic, NiFe, PVDF

\section{Introduction}

Functional and structural properties of nano-thin films differ very much to those of the same materials in bulk form. There is no fixed value thickness that defines the separation of bulk and thin film phases, but it is widely accepted that materials thicker than $100 \mathrm{~nm}$ display roughly bulk physical properties, while thinner structures begin displaying effects specific to thin films. The main contributor to these effects in thin films are the surface atoms, comprising of a larger fraction of sample volume to surface area, thus influencing its overall behaviour. Therefore, understanding the underlying processes that lead to such changes in thin films and utilizing the science that drives these changes is of great importance to modern technological applications based on thin films.

Thermal evaporation [1], pulse laser deposition [2], electro-plating [3], or plasma sputtering [4] are amongst the most popular techniques for thin film deposition. Depending on the method used, the thin film microstructure can be modified by the rate of deposition, temperature of deposition, process pressure, coating angle, or any other process conditions [5-8]. Such adjustments in coating process help to control the nucleation, adsorption of atoms to a substrate, the way they coalesce and build up subsequent layers, which leads to some intriguing results $[9,10]$. When magnetic thin films are fabricated, the above processes, as well as the in-situ application of external magnetic fields, can alter their properties during the deposition process, known as magnetic annealing [11].

Thickness of the magnetic film is also one important parameter directly responsible for the alteration of film's properties on the mesoscopic scale. For example, film thickness reduction to 
some critical level will limit the maximum grain size of a polycrystalline film, which in turn, has been linked to a modification in magnetic properties of films, such as magnetic coercive field $\left(\mathrm{H}_{c}\right)$ or its resistivity $[12,13]$.

Recent micro-magnetic simulations incorporating roughness effects have shown that the substrate roughness plays a critical role in the magnetic film's properties [14]. These studies were shortly followed by an experimental confirmation of the theoretical predictions, showing a clear correlation between the average roughness value of a substrate and the magnetic coercive field of $\mathrm{Ni}_{80} \mathrm{Fe}_{20}$ thin films [15]. As the thickness of a magnetic film decreases towards values close to the substrate roughness, the coercive field, $\mathrm{H}_{c}$, gradually increases. This was found to be in direct contrast with the grain size effect for which the $\mathrm{H}_{\mathrm{c}}$ is expected to be lower in thinner films due to a smaller grain size [16]. This remarkable and contradicting result was attributed to a change in effective magnetic anisotropy, induced by the substrate's surface roughness, as predicted theoretically [14] and later proven experimentally [17].

Although the roughness effect was previously observed experimentally in $\mathrm{Ni}_{80} \mathrm{Fe}_{20}$ Permalloy studies, it is unclear whether the same effect remains valid for other magnetic materials, or whether this is specific only to Permalloy thin films. To verify this, we have investigated two common ferromagnetic materials, Cobalt ( $\mathrm{Co}$ ) and Iron ( $\mathrm{Fe}$ ), under the same fabrication and experimental testing conditions as those used for the Permalloy. Both elements differ significantly to $\mathrm{Ni}_{80} \mathrm{Fe}_{20}$ Permalloy, which allows expanding the generality of our studies, if the roughness effect in these new materials is to be experimentally reconfirmed. Specifically, the magnetocrystalline anisotropy values $\mathrm{K}_{\mathrm{u}}$ are found to be around 22 and $31 \mathrm{~kJ} / \mathrm{m}^{3}$ for Fe and Co respectively, compared to near zero value for NiFe [18].

\section{Experiment}

Two sample sets of Fe and Co thin films were fabricated using the LabLine SPUTTER 5 magnetron plasma sputtering built by K. J. Lesker company [19]. The substrates were air-dusted before placing them into the vacuum chamber, then left in place until the base pressure reached around $1.5 \times 10^{-7}$ Torr. Instead of chemical cleaning, plasma-etching method was used in situ to clean each substrate individually for 2 minutes, using $50 \mathrm{~W}$ RF power and constant flow of Argon gas at $10 \times 10^{-3}$ Torr pressure. Samples were left in the chamber to cool under vacuum for 4 hours. All substrates were first coated with $5 \mathrm{~nm}$ seed layer of Chromium ( $\mathrm{Cr}$ ). The $\mathrm{Cr}$ layer is required to improve the adhesion between the film and substrate. We have tried depositing a selection of films without using the $\mathrm{Cr}$ seed layer and no appreciable changes in the magnetic properties / results were found. However, the films without $\mathrm{Cr}$ seed layer suffered poor adhesion and delamination. Since $\mathrm{Cr}$ presence or omission has no bearing on the results of magnetic properties reported in the manuscript, we believe the $\mathrm{Cr}$ effects are negligible in terms of roughness changes and the $\mathrm{Cr}$ under layer is applied to all our samples for improved adhesion. The sputtering target was also plasma cleaned for 2 minutes in order to remove possible oxide layer built up or contaminants on its surface. Fe and Co films of 100 and $15 \mathrm{~nm}$ thicknesses were deposited on top of the $\mathrm{Cr}$ seed layer. We selected these thicknesses because we previously observed that the surface roughness effect was negligible for 100 $\mathrm{nm}$ and maximized for $15 \mathrm{~nm} \mathrm{Ni} 80 \mathrm{Fe}_{20}$ thin films. Sputtering conditions were the following: Argon gas pressure set at $3 \times 10^{-3}$ Torr and sputtering gun kept at $60 \mathrm{~W}$ DC power. The sputtering rate for all materials was $1.9 \AA \AA \mathrm{A}$ or $11.7 \mathrm{sccm}$. All above processes took place at average temperature ranging 
between 26 to $28^{\circ} \mathrm{C}$. Extra care has been taken to maintain the same sputtering procedure and conditions for both sample sets. Magnetic thin films were fabricated using three different substrates: a) Silicon (Si) wafer (100); b) Kapton ${ }^{\circledR}$ thermal film $50 \mu$ m thick; c) $110 \mu$ m polyvinylidene fluoride (PVDF) film. These materials were chosen because of their diverse average surface roughness values $(\mathrm{Ra})$ that allow studying the roughness effect onto the magnetic properties of the final films, but also to carry out a comparative study with the previous work on $\mathrm{Ni}_{80} \mathrm{Fe}_{20}$ thin films $[15,17]$. The roughness values as measured after plasma etchings are $0.6 \mathrm{~nm}, 2.2 \mathrm{~nm}$ and $6.8 \mathrm{~nm}$ for $\mathrm{Si}$, Kapton and PVDF, respectively as reported previously [17]. All samples have the following structure: Substrate/ $\mathrm{Cr}(5 \mathrm{~nm}) /$ Ferromagnetic material $(\mathrm{t} \mathrm{nm})$, where $\mathrm{t}=15 \mathrm{~nm}$ and $100 \mathrm{~nm}$ represents the film thickness. We have intentionally omitted the use of a protective cap layer in order to facilitate high-resolution Magneto-Optic Kerr Effect (MOKE) measurements.

Magnetic properties were evaluated via MOKE measurements at room temperature under saturating external magnetic fields of up to 1000 Oe. Our experimental setup allows acquisition of a single well-defined magnetic hysteresis loop in just over a minute, without data averaging. Determination of Easy and Hard axis was achieved by measuring each sample with its long axis and sample's plane parallel to applied field, then measuring again after rotation of the sample axis by $90^{\circ}$.

\section{Results}

Figures 1. a), b) show easy axis (EA) hysteresis loops and figures 1. c), d) show the hard axis (HA) hysteresis loops for Co thin films coated on the three aforementioned substrates grouped by film thickness.

Data plots presented in EA column indicate that coercivity of Co films coated on a substrate with high average roughness value ( $\mathrm{Ra}$ ) is larger compared to the same film coated on the other two substrates having a smaller Ra. Specifically, $\mathrm{H}_{\mathrm{c}}$ for $100 \mathrm{~nm}$ thick Co film coated on PVDF rose to $152 \mathrm{Oe}$ compared to the same film coated on $\mathrm{Si}$, where the coercive field was only 105

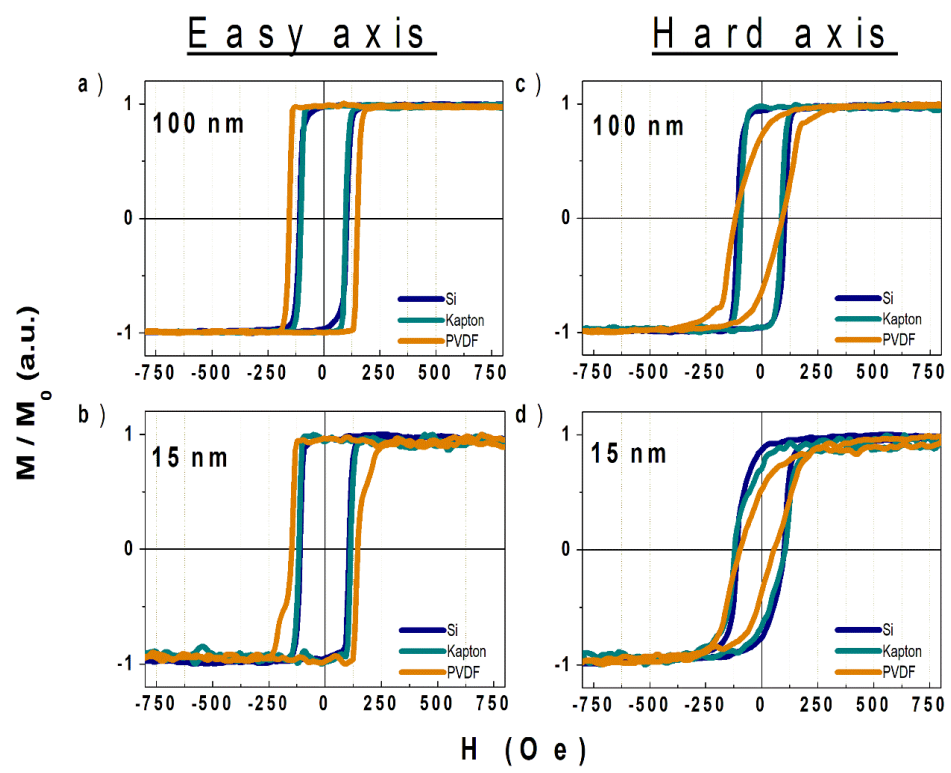

Figure 1: Hysteresis loops for 100 and $15 \mathrm{~nm}$ cobalt thin films deposited on $\mathrm{Si}$, Kapton and PVDF. Curves are grouped by film thickness and arranged into columns of easy and hard axis.

Oe. A similar result is seen on $15 \mathrm{~nm}$ film thickness where coercive field of film coated on PVDF increased to 147 Oe from 108 Oe as measured on Si substrate.

Interestingly, the HA data (Figure 1.c,d) do not indicate any significant variation of the coercive fields with the substrate roughness for both 15 and $100 \mathrm{~nm}$ Co samples. However, the effective magnetic 
anisotropy of the films appears to follow the substrate roughness sequence $\mathrm{Si}$ - Kapton - PVDF instead, with the inclination angle of the HA loops for samples on rougher substrates clearly increasing (Figure 1.c,d). The inclination was determined by measuring an angle between y-axis and imaginary line running from the origin to magnetic saturation point for each curve. For $100 \mathrm{~nm}$ thick films, we recorded $12^{\circ}, 11^{\circ}, 27^{\circ}$ angles for $\mathrm{Si}$, Kapton and PVDF respectively. Larger slope values were found for thinner $15 \mathrm{~nm}$ film with $21^{\circ}, 28^{\circ}, 35^{\circ}$ angles for the same substrates. This indicates that the observed changes are possibly related to a surface induced magnetic anisotropy, as will be discussed later in the manuscript.

Figure 2 depicts the same hysteresis data for Fe thin films. Coercive field of all films coated on Si and Kapton, tested in EA orientation, remain broadly unchanged for both $100 \mathrm{~nm}$ and $15 \mathrm{~nm}$ Fe samples (see figures 2.a, b). Noteworthy is the behaviour of the coercive field of Fe samples coated onto the roughest substrate, PVDF. The

E a s y a $x$ is $100 \mathrm{~nm}$ Fe sample coated on PVDF has a coercive field of 175 Oe, slightly larger than that of the same film coated on $\mathrm{Si}$ and Kapton with values of about 134 Oe. However, the $15 \mathrm{~nm}$ Fe thin film sample deposited onto PVDF has a remarkably larger coercive field of $256 \mathrm{Oe}$, while the coercive fields of the $15 \mathrm{~nm} \mathrm{Fe}$ films coated onto Si and Kapton substrates remain roughly constant at around 122 Oe, as clearly shown in figure 2.b.

Again, just as in the case of Co thin films, the HA measurements do not show any significant variation of the coercive fields with the substrate roughness, but they clearly show the effective magnetic anisotropy of the Fe thin films by the inclination angle of the hysteresis curves. For both $15 \mathrm{~nm}$ and $100 \mathrm{~nm}$ Fe samples, the slope of the hysteresis curves increases with the substrate roughness values. The largest slope is observed for the thinnest $15 \mathrm{~nm}$ Fe film, with the inclination angles of the loops being $12^{\circ}$ for Si substrate, $16^{\circ}$ for Kapton and $39^{\circ}$ angle from the normal for PVDF substrate, respectively.

\section{Discussion}

Thin films made of Fe and Co, prepared under the same conditions, have each responded differently to substrate roughness displaying visible and quantifiable changes in their magnetic properties. No appreciable changes were found in coercivity of Co films based on the film thickness alone (figure 1 and $3 a$ ). However, figure $3 a$ indicates a clear enhancement in $\mathrm{H}_{\mathrm{C}}$ field values attributed solely to 
roughness of the substrate, irrespective of the film thickness. In other words, a substrate with rough surface (PVDF) was found to promote larger $\mathrm{H}_{c}$ fields, unlike smooth substrate (Si) where the $\mathrm{H}_{c}$ fields are broadly unchanged.

Inclination of most hysteresis curves in HA column in figure 1, follow the trend of getting more tilted with the substrate roughness value. Interesting is the onset of this happening with respect to film thickness. The $100 \mathrm{~nm}$ thick film shows a prominent incline for PVDF only, whilst the thinner $15 \mathrm{~nm}$ films have a visible anisotropy appearing for both, PVDF and Kapton substrates. Substrate roughness promotes anisotropy in polycrystalline thin films, as proved by Sarathlal et al. by coating Co thin films on both, smooth and rippled $\mathrm{Si}$ surface. They found a smooth surface generating a feeble uniaxial anisotropy and increasing in strength with film thickness above $10 \mathrm{~nm}$ [20], however, much stronger anisotropy was witnessed in films coated on the roughened substrate.

Samples of Fe films display the roughness effect more vividly. The $15 \mathrm{~nm}$ thick Fe film coated on PVDF, the roughest substrate, recorded $46 \%$ increase in $\mathrm{H}_{\mathrm{C}}$ field, compared to the same film coated in $100 \mathrm{~nm}$ thickness (figure 2 and $3 \mathrm{~b}$ ). It is clear that the thinner the film is, the larger the increase in its coercive field value. This is in direct contradiction to some previous works concentrating on effect the grain size has on $\mathrm{H}_{\mathrm{C}}$ in which has been reported that, generally, a larger grain size, (associated with thicker films [21]), contributes to a larger coercive field $[13,16,22]$. Substrate roughness seems to directly affect the effective magnetic anisotropy (figure 2c,d). Angle of hysteresis curves, as depicted in HA column, slopes in the same manner in both thicknesses, that is, in a sequence from smooth to rough substrate. For the completeness of this study, data points for $\mathrm{Ni}_{80} \mathrm{Fe}_{20}$ thin films grown under the same conditions reported previously [15], are presented in figure 3c), which exactly follow the above finding. It is important to draw the attention that the observed trends are indeed valid, but their

a)

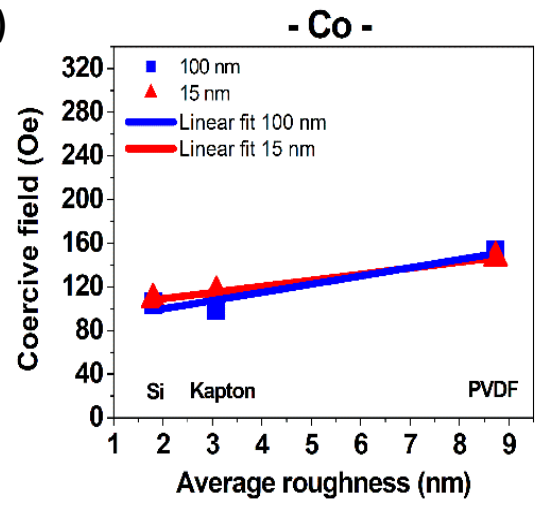

b)
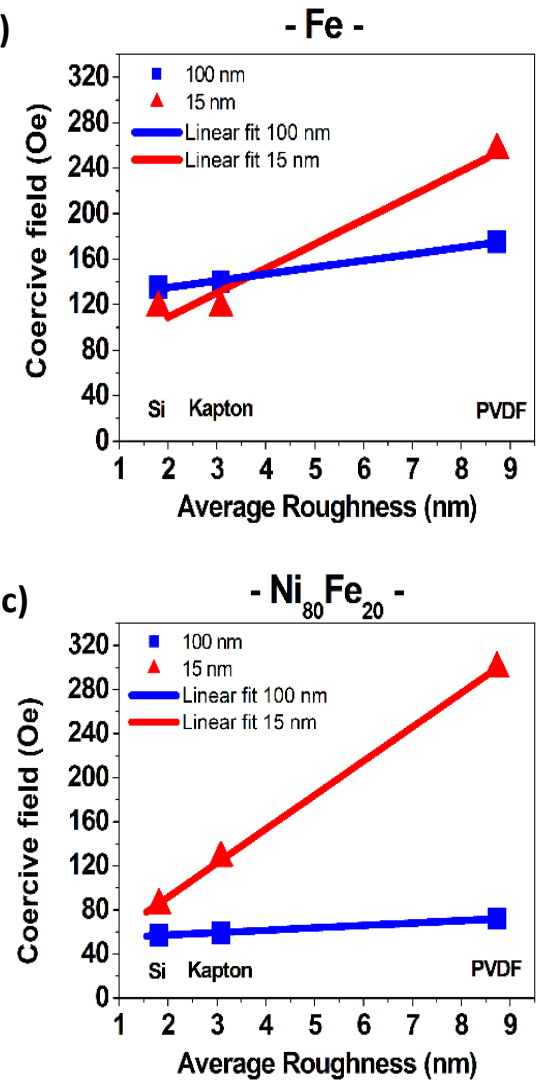

Figure 3: Coercive field values measured in easy axis sample orientation, plotted against average roughness in easy axis for $\mathrm{Co}, \mathrm{Fe}$ and NiFe. linearity is speculative as we do not have enough data points to conclude this and a non-linear dependence could occur.

From the results presented above, we see that a certain combination of substrate roughness and film thickness enhances coercivity in ferromagnetic thin films. We also see a smaller difference in $\mathrm{H}_{\mathrm{c}}$ 
values in materials having a low $\mathrm{R}_{\mathrm{a}}$ ( $\mathrm{Si}$, Kapton) that scales up with the type of material sputtered. In this study, the greatest effect was found with $15 \mathrm{~nm}$ samples.

To better emphasise these results, coercive fields of $15 \mathrm{~nm} \mathrm{Co}, \mathrm{Fe}$ and $\mathrm{Ni}_{80} \mathrm{Fe}_{20}$ films have been replotted together against substrate roughness values (figure $4 \mathrm{a}$ ). We see that $\mathrm{Co}$, having the highest $\mathrm{K}_{\mathrm{U}}$ is almost unaffected by the roughness of substrates, whilst the other two materials, Fe and $\mathrm{Ni}_{80} \mathrm{Fe}_{20}$, scale well with $\mathrm{K}_{u}$ and appear to be greatly influenced by the roughness values. We believe this is due to a surface roughness induced magnetic anisotropy as predicted by Lepadatu [14] and proven experimentally in other studies $[15,17,20]$.

Considering all anisotropy terms involved, the overall magnetic behaviour of a given magnetic thin film sample is described by an effective magnetic anisotropy, which is made up of the two main components [23]:

$$
K_{e f f}=K_{v}+\frac{K_{s}}{t}
$$

where $K_{v}$ is the volume anisotropy, a material parameter that is thickness independent, and $K_{s}$ is the surface anisotropy that has an inverse proportionality dependence to the film thickness [23]. The $\mathrm{K}_{u}$ value (i.e. $\mathrm{K}_{\mathrm{v}}$ in equation (1)) for $\mathrm{Co}$ is significantly higher than that for $\mathrm{Fe}$, while the $\mathrm{K}_{\mathrm{v}}$ value for $\mathrm{Ni}_{80} \mathrm{Fe}_{20}$ is almost negligible. Hence, according to equation (1), the surface anisotropy plays a major role in thinner films with lower $\mathrm{K}_{\mathrm{v}}$ values, as this term becomes more dominant in the effective anisotropy of magnetic thin films. This is exactly what we observed in our data for three different magnetic materials of different magneto-crystalline magnetic anisotropies, coated onto substrates of different roughness values (see figure $4 a, b$ ).

a)

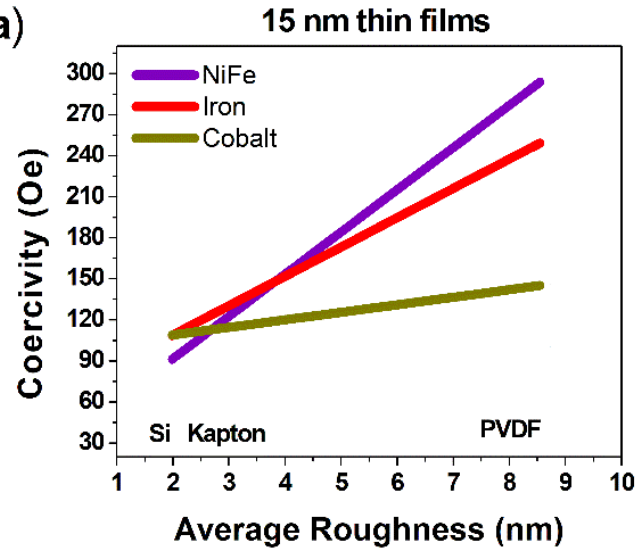

b)

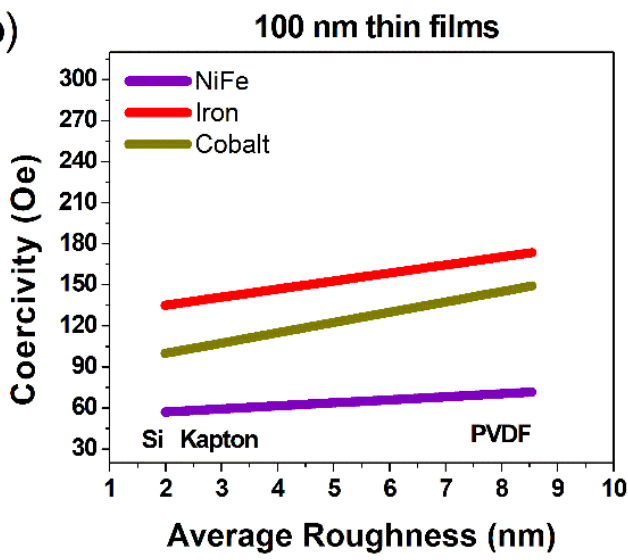

Figure 4: Coercive fields of a) - $15 \mathrm{~nm}$ and b) - $100 \mathrm{~nm}$ thin films of $\mathrm{Co}$, Fe and NiFe coated on $\mathrm{Si}$, Kapton and PVDF, plotted against substrate average roughness.

The observed results in this study cannot be explained by the grain size variations either, as our data directly contradicts previous works concentrating on the effect of the grain size on $\mathrm{H}_{\mathrm{c}}$. The coercivity of polycrystalline ferromagnetic films is well described by the random anisotropy model $[24,25]$ in which the $\mathrm{H}_{\mathrm{c}}$ variation with the grain size displays three regimes. For very small grains the $\mathrm{H}_{\mathrm{c}}$ increases rapidly with the grain size to the power six, for intermediate grain sizes the $\mathrm{H}_{c}$ is constant 
and for very large grain sizes corresponding to typically films thicker than $150 \mathrm{~nm}$, the $\mathrm{H}_{\mathrm{c}}$ decreases inverse proportionally with the grain size as [16]. Other possible effects including temperature of measurement or temperature of sample deposition are ruled out, as all our thin film fabrications and measurements took place in a temperature-controlled environment. We also made sure the experiments were carried out in such way that minimizes some other common reasons that could explain the observed effects, such as shadow deposition [26] and tensile or compressive stress $[27,28]$. Within this study, we limited our investigation to atomic force measurement of substrate roughness and we only focused on samples synthesis, magnetic properties and micromagnetics. However, an absolute confirmation would require some micro-structural measurements including XRay diffraction to check any possible lattice variations, grain sizes or crystallographic changes. In the following section, we offer further support of our results and conclusions via micromagnetics simulations.

\section{Micromagnetics simulations}

As shown in previous works on NiFe thin films $[14,15,17]$, the effect of topographical surface roughness is to induce an effective anisotropy term due to modification of the magnetostatic field. Locally, the induced anisotropy strongly depends on the roughness profile, however when averaged over the entire sample a uniaxial anisotropy contribution results, with easy axis aligned to the average roughness texture orientation. The inset in figure 5 shows a surface roughness profile obtained from an atomic force microscopy scan of a PVDF substrate, where a preferred roughness texture orientation is observed. Increasing roughness depth results in an increased induced anisotropy contribution, which leads to increased coercivity values as seen in figure 4a. On the other hand, the induced anisotropy contribution plays a smaller part for the thicker $100 \mathrm{~nm}$ films, since the induced anisotropy energy density is proportional to the roughness depth to film thickness ratio [14]. This is in agreement with the experimental observations in figure $4 b$.

For the NiFe films the change in coercivity with surface roughness depth arises solely due to the induced anisotropy contribution. With the Fe and Co films however we have the additional magnetocrystalline anisotropy contribution, where the change in coercivity with roughness depth is now given by the interaction between the induced anisotropy and the intrinsic magnetocrystalline anisotropy. In real samples the situation is complicated by the fact the easy axes of the two anisotropy contributions change relative to one another throughout the sample, partly due to the local change in the surface roughness texture, as well as due to the polycrystalline structure of the magnetic thin films. The change in coercivity in figure 4, shown for the effective easy axis direction, decreases with increasing magnetocrystalline anisotropy strength (Fe and $\mathrm{Co}$ ). In order to explain this, we calculate the change in coercivity using a micromagnetics model, which includes a surface roughness field, with Boris Computational Spintronics software [29].

The effective roughness field is computed from the topographical surface roughness profile shown in the inset to figure 5 using equation (2):

$$
\mathbf{H}\left(\mathbf{r}_{0}\right)=-\left[\sum_{\mathbf{r} \in V} \mathbf{N}\left(\mathbf{r}-\mathbf{r}_{0}\right) G\left(\mathbf{r}, \mathbf{r}_{0}\right)\right] \mathbf{M}\left(\mathbf{r}_{0}\right) \quad\left(\mathbf{r}_{0} \in V\right)
$$


Here $\mathbf{N}$ is the demagnetising tensor computed on a fine mesh with a $1 \mathrm{~nm}$ computational cellsize this mesh is fine enough to reproduce the features of the roughness profile in figure 5 . The effective roughness field $\mathbf{H}$ is proportional to the magnetisation $\mathbf{M}$ and this is obtained on a coarse computational mesh with $5 \mathrm{~nm}$ cellsize used for coercive field calculations. The function $G$ is given by:

$$
G\left(\mathbf{r}, \mathbf{r}_{0}\right)=\left\{\begin{array}{cl}
\frac{N_{V}}{N_{V r}}-1 & \mathbf{r} \wedge \mathbf{r}_{0} \in V_{R} \\
-1 & \mathbf{r} \vee \mathbf{r}_{0} \in V-V_{R}
\end{array}\right.
$$

where $N_{V}$ and $N_{V r}$ are the number of discretisation cells in the coarse and fine meshes, denoted $V$ and $V_{r}$ respectively [14]. In equation (2), in contrast to the formula given in [14] which only includes the diagonal tensor elements, we also include the off-diagonal tensor elements for completeness.

The coercive field is computed by relaxing the magnetisation configuration in an applied field using the Landau-Lifshitz-Gilbert (LLG) equation [30], which includes contributions from the demagnetising field, uniaxial magnetocrystalline anisotropy field, exchange field, applied field, as well as the roughness field. The demagnetising field is obtained using the simple Stoner-Wohlfarth model for a magnetic thin film. We still include the exchange field however, since surface roughness results in a non-uniformity of magnetisation orientation. In order to analyse the effect of different relative orientations between the magnetocrystalline anisotropy and roughness anisotropy easy axes, we simulate the two extreme cases of magnetocrystalline anisotropy easy axis parallel and perpendicular to the roughness texture orientation, as indicated in figure 5 . The results are shown in figure 5, both for $15 \mathrm{~nm}$ and $100 \mathrm{~nm}$ thick films, as a function of magnetocrystalline anisotropy strength and roughness depth.

a)

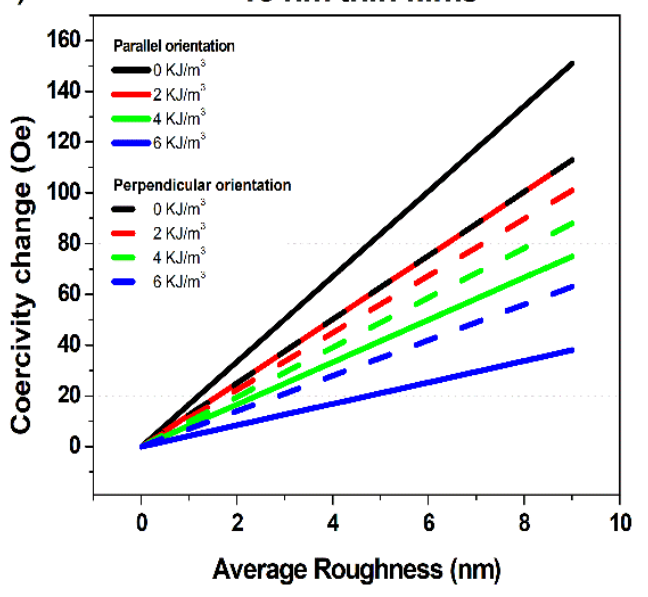

b)

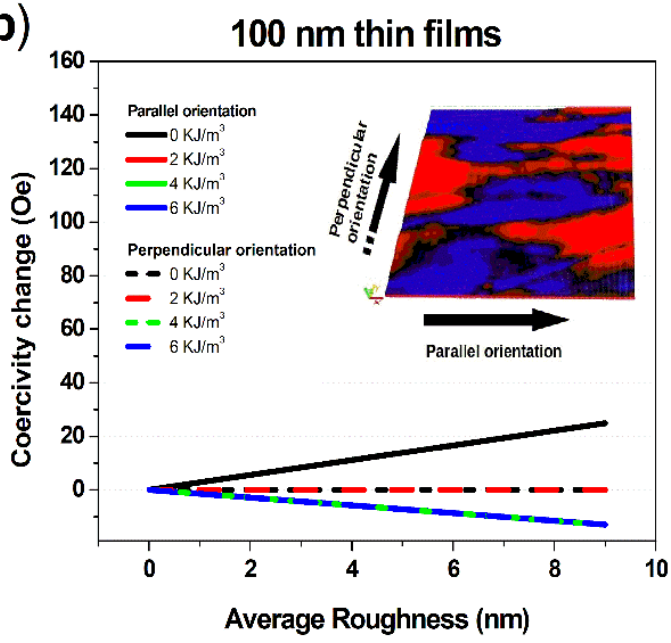

Figure 5: Coercive field change as a function of roughness depth and magnetocrystalline anisotropy strength for a) $15 \mathrm{~nm}$, and b) $100 \mathrm{~nm}$ film thickness. The inset shows a surface roughness profile, indicating the parallel (solid lines) and perpendicular (dashed lines) orientations of the magnetocrystalline anisotropy easy axis. 
For the $15 \mathrm{~nm}$ thick films, increasing the roughness depth results in increased coercivity values irrespective of the relative orientation of the magnetocrystalline anisotropy easy axis to the roughness texture, although this is most pronounced when it aligns to the roughness texture orientation. Increasing the magnetocrystalline anisotropy strength decreases this effect as the ratio of roughness to magnetocrystalline anisotropy energy density decreases. These results are in agreement with the experimental findings in figure $4 a$, indicating the reduced change in coercivity for Fe and Co arises due to increasing magnetocrystalline anisotropy energy density. For $100 \mathrm{~nm}$ thick films we again obtain a good agreement with the experimental findings in figure $4 \mathrm{~b}$, showing significantly reduced change in coercivity with roughness. These results further support the experimental findings, showing the change in coercivity for different substrates and magnetic materials can be understood as an induced effective magnetic anisotropy due to surface roughness. This work is of interest for future studies in which the roughness effect is utilized to propagate various interface roughness values in complex exchange bias systems. In fact, an increase in the interface roughness has been already reported to increase the exchange bias field and the coercive field of $\mathrm{CoO} /$ Fe systems [31,32].

\section{Conclusion}

We have prepared thin films of various thicknesses, using three common ferromagnetic materials $\mathrm{Fe}, \mathrm{Co}, \mathrm{Ni}_{80} \mathrm{Fe}_{20}$, and subjected them to magnetic measurements. The films were sputtered under identical conditions onto Si, Kapton and PVDF substrates of various roughness, allowing studying the way roughness of the substrates influences their magnetic properties. We have found that the substrate roughness, in conjunction with film thickness, is the main contributor in enhancing the magnetic coercive fields of the films as well as promoting additional magnetic anisotropy. The roughness effect becomes apparent when the value of substrate's average roughness is close to thickness of the film. Under this condition both, magnetic coercivity and anisotropy recorded largest values.

We further concluded that the type of the magnetic material also plays a significant role in observing this surface induced roughness effect, due to their different volume magneto-crystalline anisotropy contributions. $\mathrm{Ni}_{80} \mathrm{Fe}_{20}$ permalloy, which has negligible magneto-crystalline anisotropy, has been mostly influenced by the substrate roughness induced surface anisotropy, Fe with intermediate magneto-crystalline anisotropy displayed a visible but weaker response, whilst the effect in Co, which has the largest magneto-crystalline anisotropy, vanished almost completely.

Micromagnetics simulations, which take into account the topographical surface roughness, show the induced effective magnetic anisotropy arises due to a modification of the magnetostatic field. In particular, texturing of the roughness profile gives rise to an effective uniaxial anisotropy, which, in combination with the intrinsic magnetocrystalline anisotropy, explains the experimental findings on films with different magnetocrystalline anisotropy strength and substrates with different roughness depth. 


\section{Acknowledgements}

The authors gratefully acknowledge the financial support to this work received from Kurt J. Lesker Ltd and the University of Portsmouth via a PhD studentship funding.

\section{References}

[1] Y. Li, F. Li, G. Liang, W. Zheng, Y. Xu, Z. Zheng, P. Fan, Sb2Se3 thin films fabricated by thermal evaporation deposition using the powder prepared via mechanical alloying, Surf. Coat. Technol. 358 (2019) 1013-1016. doi:10.1016/j.surfcoat.2018.12.026.

[2] S.Z. Li, J. Liu, X.Z. Wang, B.W. Yan, H. Li, J. -M. Liu, Epitaxial growth of delafossite CuFeO2 thin films by pulse laser deposition, Phys. B Condens. Matter. 407 (2012) 2412-2415. doi:10.1016/j.physb.2012.03.037.

[3] C.-M. Lee, J.-H. Lim, S.-M. Hwang, E.-C. Park, J.-H. Shim, J.-H. Park, J. Joo, S.-B. Jung, Characterization of flexible copper laminates fabricated by $\mathrm{Cu}$ electro-plating process, Trans. Nonferrous Met. Soc. China. 19 (2009) 965-969. doi:10.1016/S1003-6326(08)60387-8.

[4] E. Kim, S.-W. Moon, W.-W. Park, S.-H. Han, Growth of preferred orientation Ge film using inductively coupled plasma-assisted DC magnetron sputtering at low temperature, Thin Solid Films. 548 (2013) 186-189. doi:10.1016/j.tsf.2013.09.071.

[5] R.M. Silver, E.T. Ogawa, S. Pan, A.L. de Lozanne, In-situ formation of BSCCO thin films by plasma assisted thermal evaporation, IEEE Trans. Magn. 27 (1991) 1215-1218. doi:10.1109/20.133404.

[6] D.-G. Kim, S. Lee, J.-K. Kim, Development of high density plasma assisted sputtering source for high growth rate deposition process, Curr. Appl. Phys. 11 (2011) S169-S171. doi:10.1016/j.cap.2011.03.070.

[7] C. Stegemann, R.S. Moraes, D.A. Duarte, M. Massi, Thermal annealing effect on nitrogen-doped $\mathrm{TiO} 2$ thin films grown by high power impulse magnetron sputtering plasma power source, Thin Solid Films. 625 (2017) 49-55. doi:10.1016/j.tsf.2017.01.043.

[8] R.K. Singh, N.D. Rizzo, A. Boochakravarthy, N. Newman, Large Uniaxial Anisotropy Induced in Soft Ferromagnetic Thin Films by Oblique Deposition of Underlayer, IEEE Magn. Lett. 9 (2018) 15. doi:10.1109/LMAG.2017.2779101.

[9] J. Li, X. Bi, Tailoring nucleation process to gain highly oriented $\mathrm{ZnO}$ thin film with excellent UV luminescent and electrical performances, Mater. Lett. 218 (2018) 266-269. doi:10.1016/j.matlet.2018.01.132.

[10] P.-C. Tsai, Y.-R. Jeng, Coalescence and epitaxial self-assembly of Cu nanoparticles on graphene surface: A molecular dynamics study, Comput. Mater. Sci. 156 (2019) 104-110. doi:10.1016/j.commatsci.2018.09.039.

[11] Y. Ma, G. Li, J. Du, M. Li, J. Wang, Q. Wang, Size-dependent structure and magnetic properties of co-evaporated Fe-SiO2 nanoparticle composite film under high magnetic field, AIP Adv. 6 (2016) 055929. doi:10.1063/1.4944516.

[12] Z.H. Wang, K. Chen, Y. Zhou, H.Z. Zeng, MFM studies of microstructure and magnetic properties of iron thin films prepared by sputtering, Ultramicroscopy. 105 (2005) 343-346. doi:10.1016/j.ultramic.2005.06.047.

[13] M. Vopson, M. Thwaites, G. Fernandez, S. Lepadatu, K. O'Grady, Grain size effects in metallic thin films prepared using a new sputtering technology, J. Optoelectron. Adv. Mater. 7 (2005) 2713-2720.

[14] S. Lepadatu, Effective field model of roughness in magnetic nano-structures, J. Appl. Phys. 118 (2015) 243908. doi:10.1063/1.4939093. 
[15] M.M. Vopson, J. Naylor, T. Saengow, E.G. Rogers, S. Lepadatu, Y.K. Fetisov, Development of flexible Ni80Fe20 magnetic nano-thin films, Phys. B Condens. Matter. 525 (2017) 12-15. doi:10.1016/j.physb.2017.09.005.

[16] M. Vopsaroiu, M. Georgieva, P.J. Grundy, G. Vallejo Fernandez, S. Manzoor, M.J. Thwaites, K. O'Grady, Preparation of high moment CoFe films with controlled grain size and coercivity, J. Appl. Phys. 97 (2005) 10N303. doi:10.1063/1.1855276.

[17] M. Belusky, S. Lepadatu, J. Naylor, M.M. Vopson, Evidence of substrate roughness surface induced magnetic anisotropy in Ni80Fe20 flexible thin films, J. Magn. Magn. Mater. 478 (2019) 77-83. doi:10.1016/j.jmmm.2019.01.097.

[18] E. Kita, K. Tagawa, M. Kamikubota, A. Tasaki, Magnetic recording media prepared by oblique incidence, IEEE Trans. Magn. 17 (1981) 3193-3195. doi:10.1109/TMAG.1981.1061598.

[19] Kurt J. Lesker Company | Physical Vapor Deposition Systems | Vacuum Science Is Our Business, (n.d.). https://www.lesker.com/newweb/ped/physical-vapor-deposition-systems.cfm (accessed March 25, 2018).

[20] S. K.V., D. Kumar, V. Ganesan, A. Gupta, In-situ study of magnetic thin films on nanorippled Si (100) substrates, Appl. Surf. Sci. 258 (2012) 4116-4121. doi:10.1016/j.apsusc.2011.07.105.

[21] M. Vopsaroiu, G.V. Fernandez, M.J. Thwaites, J. Anguita, P.J. Grundy, K. O textquotesingleGrady, Deposition of polycrystalline thin films with controlled grain size, J. Phys. Appl. Phys. 38 (2005) 490-496. doi:10.1088/0022-3727/38/3/022.

[22] M. Tinouche, A. Kharmouche, B. Aktaş, F. Yildiz, A.N. Koçbay, Magnetic and Structural Properties of Co Thin Films Evaporated on GaAs Substrate, J. Supercond. Nov. Magn. 28 (2015) 921-925. doi:10.1007/s10948-014-2863-y.

[23] J.M.D. Coey, Magnetism and Magnetic Materials, Cambridge University Press, Cambridge, 2010.

[24] R. Alben, J.J. Becker, M.C. Chi, Random anisotropy in amorphous ferromagnets, J. Appl. Phys. 49 (1978) 1653-1658. doi:10.1063/1.324881.

[25] G. Herzer, Grain size dependence of coercivity and permeability in nanocrystalline ferromagnets, IEEE Trans. Magn. 26 (1990) 1397-1402. doi:10.1109/20.104389.

[26] N. Chowdhury, S. Mallick, S. Mallik, S. Bedanta, Study of magnetization relaxation in Co thin films prepared by substrate rotation, Thin Solid Films. 616 (2016) 328-334. doi:10.1016/j.tsf.2016.08.043.

[27] D. Kumar, S. Singh, P. Vishawakarma, A.S. Dev, V.R. Reddy, A. Gupta, Tailoring of in-plane magnetic anisotropy in polycrystalline cobalt thin films by external stress, J. Magn. Magn. Mater. 418 (2016) 99-106. doi:10.1016/j.jmmm.2016.03.072.

[28] V. Shukla, C. Mukherjee, R. Chari, S. Rai, K.S. Bindra, A. Banerjee, Uniaxial magnetic anisotropy of cobalt thin films on different substrates using CW-MOKE technique, J. Magn. Magn. Mater. 370 (2014) 100-105. doi:10.1016/j.jmmm.2014.06.061.

[29] Boris Computational Spintronics - Boris Micromagnetics, (n.d.). https://www.borisspintronics.uk/ (accessed June 17, 2019).

[30] W.F. Brown, Micromagnetics, J. Wiley, New York; London, 1963.

[31] C. Fleischmann, F. Almeida, J. Demeter, K. Paredis, A. Teichert, R. Steitz, S. Brems, B. Opperdoes, C. Van Haesendonck, A. Vantomme, K. Temst, The influence of interface roughness on the magnetic properties of exchange biased CoO/Fe thin films, J. Appl. Phys. 107 (2010) 113907. doi:10.1063/1.3391470.

[32] D. Kumar, S. Singh, A. Gupta, Effect of interface roughness on exchange coupling in polycrystalline $\mathrm{Co} / \mathrm{CoO}$ bilayer structure: An in-situ investigation, J. Appl. Phys. 120 (2016) 085307. doi:10.1063/1.4961521. 